\title{
Evidence for a Low-Compressibility Carbon Nitride Polymorph Elaborated at Ambient Pressure and Mild Temperature
}

Graziella GOGLIO ${ }^{1, *}$, Denis $\mathrm{FOY}^{1}$, Stanislav PECHEV ${ }^{1}$, Jérôme MAJIMEL ${ }^{1}$, Gérard DEMAZEAU ${ }^{1}$, Nicolas GUIGNOT ${ }^{2}$, Denis ANDRAULT ${ }^{3}$

${ }^{1}$ CNRS, Université Bordeaux, ICMCB, 87 avenue du Dr. A. Schweitzer, 33608 PESSAC, France

2 European Synchrotron Radiation Facilities, Grenoble, France. Now at Synchrotron Soleil, Saint-Aubin - BP 48, 91192 GIF-sur-YVETTE Cedex, France

${ }^{3}$ Laboratoire des Magmas et Volcans, UMR6524, Université Blaise Pascal, 5 rue Kessler, 63000 CLERMONT-FERRAND, France

* Corresponding author: Graziella GOGLIO

Email : goglio@icmcb-bordeaux.cnrs.fr

Fax : +33 540002761

Tel : +33540006334

Postal Address : CNRS-ICMCB, Université de Bordeaux, 87 avenue du Dr. A. Schweitzer, 33608 Pessac, France 


\section{ABSTRACT}

Superhard materials like diamond are essential for abrasive or cutting tool applications. In this way, carbon nitrides are of relevant interest because they are expected to exhibit exceptional mechanical properties, high values of bulk modulus being predicted. A smart and simple method was used to synthesize carbon nitrides and allowed elaborating a low-compressibility polymorph. The processing consists in the decomposition of commercial thiosemicarbazide $\left(\mathrm{H}_{2} \mathrm{NC}(\mathrm{S}) \mathrm{N}_{2} \mathrm{H}_{3}\right)$ powder at ambient pressure and $600^{\circ} \mathrm{C}$ under nitrogen flow. Besides the presence of a graphitic $\mathrm{C}_{3} \mathrm{~N}_{4}$ phase, a nanocrystalline material is obtained and is identified as a cubic carbon nitride with a cell parameter of $3.163 \AA$. Its impressive bulk modulus of $355 \mathrm{GPa}$ propels it as a challenger for cubic boron nitride among lowcompressibility materials. Thus, we prove that, in accordance with previous numerous theoretical predictions, crystalline carbon nitrides exhibit exceptional compressibility behaviour. Moreover, our synthesis strategy evidences that, despite usual admitted considerations, severe and expensive pressure and temperature conditions are not mandatory to elaborate low-compressibility covalent materials, which could give new insights for their development.

Keywords : Carbon Nitride; Nanocrystalline; High Resolution Electron Microscopy; Mechanical Properties; Bulk Modulus; Equation of State 


\section{Introduction}

Considering that the nature of the bonding and arrangement of atoms in a solid determine its mechanical properties, dense covalent materials are expected to exhibit the highest superhardnesses, especially those consisting of elements from the B-C-N triangle. Then, because it is the hardest material of all known solids, diamond appears fascinating and is currently used for cutting, drilling, grinding, or polishing tools in industry [1]. Cubic boron nitride (c-BN) ranks second among superhard materials and is widely used as abrasive [2]. Recently, $\mathrm{BC}_{2} \mathrm{~N}$ heterodiamond was synthesized and found to exhibit an unusual combination of mechanical parameters as its bulk modulus is lower than that of c-BN while its Vickers hardness ranks second only after that of diamond [3]. Since 1989, the C-N binary system also aroused deep interest because exceptional mechanical properties were theoretically predicted for three-dimensional (3D) $\mathrm{C}_{3} \mathrm{~N}_{4}$ by Liu and Cohen [4]. Consequently many efforts were devoted to the experimental and theoretical studies of highly incompressible carbon nitrides $\left(\mathrm{CN}_{\mathrm{x}}\right)$. $A b$ initio calculations first proposed several crystallographic networks (either hexagonal or cubic) for dense $\mathrm{CN}_{\mathrm{x}}$ with $\mathrm{C}_{3} \mathrm{~N}_{4}$ composition where carbon and nitrogen are in tetrahedral $\left(\mathrm{C} \mathrm{sp}^{3}\right)$ and trigonal $\left(\mathrm{N} \mathrm{sp}^{2}\right.$ or $\left.\mathrm{sp}^{3}\right)$ coordinations, respectively [4-8]. According to calculations, bulk moduli $\mathrm{B}$ of $3 \mathrm{D} \mathrm{C}_{3} \mathrm{~N}_{4}$ polymorphs are expected to reach and even exceed (when nitrogen adopts $\mathrm{sp}^{3}$ hybridization) diamond value. Further calculations also suggested that the 3 to 4 stoichiometric $C / N$ ratio $\left(C_{3} N_{4}\right)$ may not be necessary to obtain very low compressibility in these 3D networks [9- 
12]. For example, dense carbon nitrides with $\mathrm{CN}$ composition where $\mathrm{C}$ and $\mathrm{N}$ both adopt $\mathrm{sp}^{2}$ hybridization could exhibit bulk moduli in the range 345-375 GPa [9]. These values are comparable with those predicted for their pure carbon homologues similar in structure, while higher than for the $\mathrm{BN}$ ones where bond is less covalent. Then, because $\mathrm{C}-\mathrm{N}$ distance is particularly short and $\mathrm{C}-\mathrm{N}$ chemical bond is strongly covalent, 3D carbon nitrides are expected to exhibit superhardness properties.

For this reason, many attempts were carried out to synthesize these materials $[13,14]$. Because, until now, the most superhard materials such as diamond, $\mathrm{c}-\mathrm{BN}$ or even $\mathrm{BC}_{2} \mathrm{~N}$ require severe pressure and temperature conditions to be elaborated, high-pressure high-temperature processes were widely used in the case of carbon nitrides. Many other techniques including solvothermal reactions, mechanosynthesis, use of shockwaves, thermal decomposition of triazines, thin films elaboration were also performed for crystalline $\mathrm{CN}_{\mathrm{x}}$ synthesis attempts $[13,14]$. Experimental evidences for hexagonal structures of $\mathrm{C}_{3} \mathrm{~N}_{4}$ (most of the results concerning crystalline carbon nitride nanoclusters embedded in amorphous matrix) as well as other unpredicted 3D structures with unknown compositions were reported. These works underlined the great difficulty to retain nitrogen in the material, to obtain crystallized carbon nitrides and to stabilize 3D networks [13].

Nevertheless, despite some dense $\mathrm{CN}_{\mathrm{x}}$ have been claimed (mainly with $\beta$ $\mathrm{C}_{3} \mathrm{~N}_{4}$ structure or unidentified networks $\left.[13,15]\right)$, their compressibility features and 
bulk modulus have not been established until now. Recently, a bulk modulus of 277GPa was measured in a carbon nitride imide $\mathrm{C}_{2} \mathrm{~N}_{2}(\mathrm{NH})$ elaborated under severe conditions [16]. Although high, this value was suspected to be lower than those predicted for dense $\mathrm{C}_{3} \mathrm{~N}_{4}$ polymorphs because of the presence of $\mathrm{N}-\mathrm{H}$ groups that do not contribute to the solid-state network. So the high mechanical performances of carbon nitrides remain an opened and fascinating question.

We report here the elaboration and discovery of an unpredicted lowcompressibility carbon nitride. It has been synthesized in very soft experimental conditions (ambient pressure and mild temperature) far from those usually required for low-compressibility covalent materials. Moreover, its high incompressibility propels it as a direct rival for cubic boron nitride.

\section{Experimental section}

In the typical procedure, 2 grams of thiosemicarbazide $\left(\mathrm{H}_{2} \mathrm{NC}(\mathrm{S}) \mathrm{N}_{2} \mathrm{H}_{3}\right.$ Aldrich, 99\%) were placed in a Pyrex boat, this manipulation being performed in a glove-box with flowing nitrogen gas because the organic reagent is hygroscopic. The tube was maintained during any transport under $\mathrm{N}_{2}$ atmospher and was introduced in a tubular furnace under nitrogen flow. Temperature was slowly increased $\left(1^{\circ} \mathrm{C} / \mathrm{min}\right)$ up to several consecutive intermediate $1 \mathrm{~h}$ steps at 180,250 , 350 and $480^{\circ} \mathrm{C}$ and then finally maintained at $600{ }^{\circ} \mathrm{C}$ during $10 \mathrm{~h}$. Then the sample 
was cooled down to room temperature under nitrogen flow $\left(1^{\circ} \mathrm{C} / \mathrm{min}\right)$. The measured mass loss was about $68 \%$.

The as-obtained powder was dispersed in alcohol by ultrasonication and a drop of the suspension was deposited on a copper grid covered with a carbon film or on an aluminium pin for TEM or SEM experiments, respectively. The preparation was finally air-dried. It should be noticed that the use of a carbon membrane was necessary to retain the nanoparticules on the copper grid.

High resolution transmission electron microscopy (HRTEM) and energy filtered transmission electron microscopy (EFTEM) observations were performed using a field emission gun JEOL 2200 FS operating at 200kV, equipped with an in-column filter (Omega Filter). The spatial resolution is $0.23 \mathrm{~nm}$. High resolution images were acquired with a Gatan Ultrascan CCD $2 \mathrm{k} \times 2 \mathrm{k}$. Digital Diffractograms were calculated using the Gatan Digital Micrograph program. The EFTEM maps were obtained by the three window method on the K edge (401 eV) of Nitrogen.

Scanning electron microscopy (SEM) and energy dispersive X-ray spectroscopy (EDXS) experiments were performed on a JEOL 840A and a JEOL 6700 s (field emission gun) operating both at $10 \mathrm{kV}$, and with energy resolutions of $136 \mathrm{eV}(\mathrm{Mn})$ and $131 \mathrm{eV}(\mathrm{Mn})$, respectively.

Bulk elemental combustion analysis of $\mathrm{C}, \mathrm{H}, \mathrm{N}, \mathrm{O}, \mathrm{S}$ elements was performed on a Thermofisher Flash EA 112 analyzer.

Powder X-ray diffraction (XRD) experiments were performed at 1 bar and at high pressure at the ID27 beamline at ESRF. A gas-driven membrane diamond anvil cell (DAC) was used for the in-situ measurements from atmospheric pressure up to 
40GPa. For compressibility measurements, the sample was loaded between two $\mathrm{NaCl}$ pellets in a cavity drilled in a rhenium gasket. $\mathrm{NaCl}$ acted as both pressure transmitting medium and pressure calibrant. To measure pressure into the DAC, we used the equations of state (EOS) of the $\mathrm{NaCl} \mathrm{B1} \mathrm{[17]} \mathrm{or} \mathrm{B2} \mathrm{[18]} \mathrm{forms} \mathrm{above}$ or below $\sim 30 \mathrm{GPa}$. To validate this pressure scale, pure diamond grains $(0.3 \mu \mathrm{m}$ grain size) were inserted simultaneously with $\mathrm{c}-\mathrm{CN}_{\mathrm{x}}$ and $\mathrm{NaCl}$ into the DAC in order to determine diamond's EOS and compare it with currently admitted values $[1,19]$. This simultaneous determination of diamond's EOS also allowed a quantitative comparison with our superhard carbon nitride phase. Some samples were laserheated at high pressure in the DAC using a YAG infrared laser [20,21]. For experiments at atmospheric pressure, the DAC was opened to allow collecting some of the high-angle diffraction peaks, the sample being simply placed into the

cavity drilled in the rhenium gasket. Angle dispersive $(\lambda=0.3738 \AA)$ diffraction images were captured on a Mar345 plate detector and integrated using the FIT2D software [22] The whole pattern profile analysis of the powder diffraction data was performed using the Fullprof program [23].

\section{Results and discussion}

To prevent nitrogen departure during carbon nitride elaboration, we performed the synthesis at moderate temperature using precursors of high reactivity, the thermal decomposition of an organic reagent (thiosemicarbazide) 
being chosen as the synthesis route. After thermal treatment completed, chemical analysis by elemental combustion of the as-obtained orange powder leads to 35 weight- $\%$ carbon, 56.8 weight- $\%$ nitrogen, 1.9 weight- $\%$ hydrogen and 6.3 weight$\%$ oxygen. No sulfur is present in the obtained material (thermal analysis coupled with mass spectrometry has evidenced that its departure from the chemical system during decomposition is mainly due to the formation of $\mathrm{H}_{2} \mathrm{~S}$ [26]), sulfur can then be totally excluded as a potential contaminant.

The structural characterization of the synthesized material under synchrotron radiation allows to observe two types of XRD patterns when probing different sample portions. The first one mainly consists of a broad reflection at 3.24 $\AA$ characteristic of an highly hydrogenated graphitic carbon nitride that we previously characterized combining EELS [25], XRD, IR, TGA -MS, XPS and NMR analysis [26]. In this graphitic material, we evidenced the presence of water molecules either adsorbed on the surface or trapped into the voids of the graphene planes, which fully explains a part of hydrogen amount and the presence of oxygen (which is not bonded neither to carbon nor to nitrogen) in the system [26]. The second type of XRD pattern appears more complex and can be fully indexed on the basis of a cubic unit cell with $a=3.163 \pm 0.002 \AA$ (see Le bail fit in Fig. 1). This diffraction pattern can not be attributed to any predicted carbon nitride neither with $\mathrm{C}_{3} \mathrm{~N}_{4}$ nor with $\mathrm{CN}$ composition [4-12]. A careful examination of the visible Bragg line indexes indicates one single reflection condition for $h k l$ values: $h+k+I=2 n$ which is indicative of a I-centred lattice. The presence of reflections (200) or (310) excludes any additional conditions on $h k 0$ or $h 00$. Thus, the possible space groups 
are limited to: 123 (197), $12{ }_{1} 3$ (199), Im-3 (204), I432 (211), I-43m (217), Im-3m (229). The powder diffraction results do not allow further structural analysis and determination of an unambiguous model for the atomic site occupation. Moreover, because of the low amount of sample placed into a diamond anvil cell, the XRD patterns can not be regarded as representative of the whole material and are then not significant enough to quantify accurately the relative amount of graphitic and cubic phases. However, some few percents can be admitted as rough estimation of the cubic phase amount.

To improve characterization of this cubic phase, HRTEM experiments were carried out on the sample and reveal coexistence of two kinds of particles. Besides numerous micrometric grains of graphitic carbon nitride [24-26], smaller particles with nanometric size $(20-50 \mathrm{~nm})$ are widely present. In fact, they can be described as nanosized crystalline domains of about $5 \mathrm{~nm}$ embedded into an amorphous matrix (Fig. 2). This matrix happens to be unstable under electron beam irradiation and quickly collapses, which favours observation of the nanodomains crystallographic planes. Conversely, this crystalline phase is not affected by electron beam irradiation. Digital diffractograms analysis ensures that these crystalline nanodomains correspond to the I-centred cubic phase evidenced by XRD (the reticular distances $2.2 \AA$ and $1.6 \AA$ correspond to the (110) and (200) reflections of the I-centred cubic lattice, respectively ; the cell parameter value is then equal to $3.1 \pm 0.1 \AA$ ). This unambiguously proves that the cubic phase is synthesized during the soft thermal decomposition process and not induced by the electron beam irradiation during the HRTEM experiment. To elucidate the chemical 
composition of the cubic phase, chemical analysis of the whole $20-50 \mathrm{~nm}$ particles was carried out by EDXS in scanning electron microscope, therefore at a scale that includes both nanosized crystalline domains and amorphous matrix. It reveals presence of both carbon and nitrogen with significant contents and no other element is detected. Moreover the EFTEM analyses clearly evidence that nitrogen is present into the cubic nanodomains rather than into the amorphous matrix (Fig. 3). Unfortunately, carbon could not be measured by EFTEM owing to the presence of carbon film on the copper grid used for the experiment, this carbon membrane being necessary to retain the nanoparticles on the grid. Nevertheless, as only C and $\mathrm{N}$ were detected in the $20-50 \mathrm{~nm}$ particles, and considering that nitrogen alone is gaseous at ambient pressure, there is little doubt that the cubic phase is a carbon nitride $\left(\mathrm{c}-\mathrm{CN}_{\mathrm{x}}\right)$. Nevertheless, it must be mentioned that the presence of hydrogen can not be excluded. One should also notice that our experimental procedure to prepare samples for TEM and SEM characterizations was optimized to observe nanometric particles ; in this way, it is not possible to quantify accurately the relative amount of graphitic and nanometric phases on the basis of microscopy observations.

At this stage, it is then clear that a 3D carbon nitride has been synthesized despite weak pressure and temperature conditions involved. The main question then concerns its mechanical performances. Because bulk modulus is a sensitive indicator of hardness in light element-based materials $[27,28]$, we investigated the high pressure properties of the new $c-\mathrm{CN}_{\mathrm{x}}$ phase using in situ XRD in a DAC. Up to $40 \mathrm{GPa}$, the persistence of the diffraction pattern and absence of additional peaks 
indicates that neither structural phase transition nor chemical decomposition occurred. Moreover, no particular change of the diffraction pattern was observed after laser heating at $2000 \mathrm{~K}$ for a pressure of $20 \mathrm{GPa}$, indicating that $\mathrm{c}-\mathrm{CN}_{\mathrm{x}}$ remains stable in such severe conditions. The isothermal bulk modulus $\left(B_{0}\right)$ and its first pressure derivative $\left(\mathrm{B}^{\prime}{ }_{0}\right)$ were derived from $\mathrm{P}-\mathrm{V}$ measurements performed at $300 \mathrm{~K}$ based on the third-order Birch-Murnaghan equation of state (EOS) "P(V) $=3 / 2 B_{0}\left[\xi^{7 / 3}-\xi^{5 / 3}\right]\left[1+3 / 4\left(B_{0}^{\prime}-4\right)\left(\xi^{2 / 3}-1\right)\right]^{\prime \prime}$ where $\xi=V_{0} / V$ with $V_{0}$ and $V$ the volumes at ambient and $P(V)$ pressures, respectively (Fig. 4) [29]. First of all, we verify that, given the experimental uncertainties, the refined values of $\mathrm{V}_{0}=45.50 \pm 0.02 \AA^{3}, \mathrm{~B}_{0}=460 \pm 12 \mathrm{GPa}$ and $\mathrm{B}_{0}{ }_{0}=4.1 \pm 0.7$ for diamond are compatible with published values, which validates our pressure scale (if $\mathrm{B}_{0}^{\prime}$ is fixed to 4 , the refined value of $B_{0}$ is $462 \pm 4 \mathrm{GPa}$ for diamond) $[1,19]$. Concerning the $c-\mathrm{CN}_{\mathrm{x}}$ phase, we obtain $\mathrm{B}_{0}=355 \pm 11 \mathrm{GPa}$ for $\mathrm{B}_{0}{ }_{0}=3.2 \pm 0.6$, the latter being consistent with values predicted for carbon nitrides and for solids in general $[7,8]$; the refined $V_{0}$ corresponds to $a=3.165 \pm 0.003 \AA$ (Fig. 4). If we maintain $B_{0}^{\prime}$ to a fixed value of 4.0 , the experimental value determined for C-BN [2] and usually used to compare solids between each others, we obtain $\mathrm{B}_{0}=337 \pm 4 \mathrm{GPa}$ for $\mathrm{c}-\mathrm{CN}_{\mathrm{x}}$. Thus, the bulk modulus of the cubic carbon nitride appears remarkably high with a compressibility equivalent to $73 \%$ and $91 \%$ of diamond (this work) and c-BN [2] compressibilities, respectively. Moreover, its performances appear better than the low-density cubic $\mathrm{BC}_{2} \mathrm{~N}$ phase and comparable with this heterodiamond high-density polymorphs recently claimed (all $\mathrm{BC}_{2} \mathrm{~N}$ polymorphs being elaborated under high pressure and 
temperature) [3, 30-31]. Finally, despite the fact that $\mathrm{c}-\mathrm{CN}_{\mathrm{x}}$ exhibits a bulk modulus lower than theoretical values calculated for dense $\mathrm{C}_{3} \mathrm{~N}_{4}$ polymorphs, its very low compressibility remains impressive and of the same order than values predicted for $\mathrm{CN}$ phases involving $\mathrm{sp}^{2}$ bonds [9]. When comparing with $\mathrm{C}_{2} \mathrm{~N}_{2}(\mathrm{NH})$, we observe a higher bulk modulus [16]. This difference can originate either from the carbon nitride network itself (it then should be more dense in our case), or the presence of hydrogen (which tends to decrease B values as mentioned by Horvath-Bordon [16], the hydrogen presence being not excluded in our case), or the nanometric size of our material. In this last case, it is difficult to predict if a bulk morphology for $\mathrm{c}-\mathrm{CN}_{\mathrm{x}}$ should favor or not higher values of bulk modulus as both tendancies can be encountered in literature [32-35]. Moreover, the nanocomposite morphology (the nanosized cubic phase being embedded in an amorphous nanosized matrix) makes these predictions all the more difficult. However, in the most probable scenario, the amorphous matrix has a higher compressibility than the nanocrystals. Therefore, the amorphous matrix can accommodate and relax the strains ; in other words, the surrounding isotropic matrix distributes the impact of pressure to the matrix-nanocrystallite interface, acting then as a hydrostatic pressure transmitting medium [36-37]. An accurate determination of the interface between $\mathrm{c}-\mathrm{CN}_{\mathrm{x}}$ and the matrix should then be necessary in further works, because it can for example provide information to explain the stabilty of the nanoclusters.

The diffraction results could provide another clue to estimate $\mathrm{c}-\mathrm{CN}_{\mathrm{x}}$ composition. For this, we should first consider the $N_{\text {at }} / N_{0}$ ratios for diamond and c$B N$, where $N_{a t}$ is the number of atoms per unit cell. Diamond $\left(V_{0}=45.38 \AA^{3}, N_{a t}=\right.$ 
8) [38] and C-BN $\left(V_{0}=47.28 \AA^{3}, N_{a t}=8\right)$ [39] present $N_{a t} / N_{0}$ ratios of 176 atom $/ \mathrm{nm}^{3}$ and 169 atom $/ \mathrm{nm}^{3}$, respectively. For various phases in the carbonnitrogen system, theoretical calculations predict $N_{a t} N_{0}$ ratios ranging from 158 to 179 atom $/ \mathrm{nm}^{3}$, depending on the considered material structure and composition [49]. As C-CN $N_{x}$ exhibits a unit cell volume $V_{0}=31.64 \pm 0.06 \AA^{3}$, its $N_{a t} / N_{0}$ ratio should be equal to 158 or $190 \AA^{3} /$ atom if the unit cell contains 5 or 6 atoms, respectively. The value $\mathrm{N}_{\mathrm{at}}=6$ appears unlikely because it would correspond to a carbon nitride phase more dense than diamond and c-BN, which should lead to an even more incompressible phase than measured in this study. Thus, we can make the hypothesis that the unit cell probably contains five atoms, and reasonably assign to the new carbon nitride formula such as $\mathrm{C}_{y} \mathrm{~N}_{5-\mathrm{y}}$ with y between 1 and 4 (or $\mathrm{C}_{\mathrm{y}} \mathrm{N}_{5-\mathrm{y}}$ ${ }_{\delta} \mathrm{H}_{\delta}$ with $\left.1<(\mathrm{y}, \delta)<4\right)$.

\section{Conclusion}

As expected by theoretical predictions, we have shown here that 3D carbon nitrides can be incompressible as evidenced by $\mathrm{c}-\mathrm{CN}_{\mathrm{x}}$ impressive bulk modulus. Surprisingly, $\mathrm{c}-\mathrm{CN}_{\mathrm{x}}$ has been stabilized under pressure and temperature conditions much less severe than those usually required for superhard covalent materials syntheses. Improving its synthesis could give rise to increase its amount (it is here only present in few percents), to enhance its crystallite size and then to favor its characterization, especially concerning its accurate chemical composition and 
structure. Special efforts should also be given to understand involved phenomena at $180^{\circ} \mathrm{C}$ during decomposition as, at this temperature, the system is liquid and undergoes the first mass loss. The morphology obtained here (crystallized phase embedded into amorphous matrix) could perharps be correlated to this liquid state step.

However, one should consider that nowadays one of the strategies in the search for superhard materials is the elaboration of new forms of diamond [40]. In particular, nanocrystalline diamond produced by high-pressure high-temperature process appears as hard as (or even harder than) single-crystal diamond [35]. As, contrary to $\mathrm{c}-\mathrm{CN}_{\mathrm{x}}$, the amorphous matrix embedding the crystalline nanodomains is highly unstable, further works to isolate and disperse the nanoclusters deserve careful thought. The production of primary incompressible particles with $5 \mathrm{~nm}$ in diameter could then provide $\mathrm{c}-\mathrm{CN}_{\mathrm{X}}$ as a raw material for composites elaboration for example.

\section{Acknowledgments}

Authors thank the Centre National de la Recherche Scientifique for his support ((Programme Matériaux Nouveaux - Fonctionnalités Nouvelles), Dr Mohammed Mezouar (ID27 beamline) from European Synchrotron Radiation Facilities (ESRF, France), Elisabeth Sellier and the Centre de Ressources en Microscopie Electronique et Microanalyse (CREMEM) at University of Bordeaux 1 for microscopy experiments. Prof. Olivier Chauvet, Prof. Michel Pouchard and Prof. 
Goglio et al., revised version submitted to Diamond and Related Materials

Jean Paul Issi are gratefully acknowledged for fruitful discussions. 
Goglio et al., revised version submitted to Diamond and Related Materials

\section{References}

1. F. Ocelli, P. Loubeyre, R. Le Toullec, Nature Materials 2 (2003) 151

2. E. Knittle, R.M. Wentzcovitch, R. Jeanloz, M.L. Cohen, Nature 337 (1989) 349

3. V.L. Solozhenko, D. Andrault, G. Fiquet, M. Mezouar, D.C. Rubie, Appl. Phys. Lett. 78 (2001) 1385

4. A.Y. Liu, M.L. Cohen, Science 245 (1989) 841

5. A. Y. Liu, R.M. Wentzcovitch, Phys. Rev. B 50 (1994) 10362

6. Y. Guo, W.A. Goddard, Chem. Phys. Lett. 237 (1995) 72

7. D.M. Teter, R.J. Hemley, Science 271 (1996) 53

8. J. Martin-Gil, F.J. Martin-Gil, M. Sarikaya, M. Qian, M. José-Yacaman, J. Appl. Phys. 81 (1997) 2555

9. M. Côté, M.L. Cohen, Phys. Rev. B 55 (1997) 5684

10. E. Sandré, C.J. Pickard, C. Colliex, Chem. Phys. Lett. 325 (2000) 53

11. R. Weihrich, S.F. Matar, E. Betranhandy, V. Eyert, Solid State Sci. 5 (2003) 701

12. E. Betranhandy, S.F. Matar, Diamond Relat. Mater. 15 (2006) 1609

13. G. Goglio, D. Foy, G. Demazeau, Mater. Sci. Eng. R 58 (2008) 195

14. S. Muhl, J.M. Mendez, Diamond Relat. Mater. 8 (1999) 1809

15. J. Liu, T. Sekine, T. Kobayashi, Solid State Commun. 137 (2006) 21 
Goglio et al., revised version submitted to Diamond and Related Materials

16. E. Horvath-Bordon, R. Riedel, P.F. McMillan, P. Kroll, G. Miehe, P.A. Van Aken, A. Zerr, P. Hoppe, O. Shebanova, I. McLaren, S. Lauterbach, E. Kroke, R. Boehler, Angew. Chem. Int. Ed. 46 (2007) 1476

17. J.M. Brown, J. Appl. Phys. 86 (1999) 5801

18. N. Sata, G. Shen, M.L. Rivers, S.R. Sutton, Phys. Rev. B 65 (2002) 104114

19. K. Kunc, I. Loa, K. Syassen, High Press. Res. 24 (2004) 101

20. D. Andrault, G. Fiquet, Rev. Sci. Instr. 72 (2001) 1283

21. E. Schultz, M. Mezouar, W. Crichton, S. Bauchau, G. Blattmann, D. Andrault, G. Fiquet, R. Boehler, N. Rambert, B. Sitaud, P. Loubeyre, High Press. Res. 25 (2005) 71

22. A. Hammersley (1995) Program FIT2D, European Synchrotron Radiation Facilities, Grenoble, France

23. J. Rodriguez-Carvajal, Newsletter of the powder diffraction commission of the international union of crystallography 26 (2001) 12

24. G. Goglio, D. Andrault, S. Courjault, G. Demazeau, High Press. Res. 22 (2002) 535

25. P. Moreau, F. Boucher, G. Goglio, D. Foy, V. Mauchamp, G. Ouvrard, Phys. Rev. B 73 (2006) art. 195111

26. D. Foy, G. Demazeau, P. Florian, D. Massiot, C. Labrugère, G. Goglio, accepted for publication in J. Solid State Chem. 
Goglio et al., revised version submitted to Diamond and Related Materials

27. M.L. Cohen, Mat. Sci. Eng. A 105-106 (1988) 11

28. M.L. Cohen, J. Hard Mater. 2 (1991) 13

29. F. Birch, J. Geophys. Res. 83 (1978) 1257

30. Y. Zhao, D.W. He, L.L Daemen, T.D. SHen, R.B. Schwarz, Y. Zhu, D.L. Bish, J. Huang, J. Zhang, G. Shen, J. Qian, T.W. Zerda, J. Mater. Res. 17 (2002) 3139

31. E. Kim, T. Pang, W. Utsumi, V.L. Solozhenko, Y. Zhao, Phys. Rev. B 75 (2007) art. 184115

32. A. Latapie, D. Farkas, Scripta Mater. 48 (2003) 611

33. K. Lin, J. Chang, J. Lu, W. Wu, Y. Zeng, Ceram. Int. 33 (2007) 979

34. S.-J. Zhao, K. Albe, H. Hahn, Scripta Mater. 55 (2006) 473

35. T. Irifune, A. Kurio, S. Sakamoto, T. Inoue, H. Sumiya, Nature 421 (2003) 599

36. K.E. Lipinska-Kalita, P.E. Kalita, O.A. Hemmers, T. Hartmann, Phys. Rev. B 77 (2008) art. 094123

37. K.E. Lipinska-Kalita, P.E. Kalita, C. Gobin, O.A. Hemmers, T. Hartmann, G. Mariotto, Phys. Rev. B 77 (2008) art. 134107

38. H.J. Grenville-Wells, K. Lonsdale, Nature 181 (1958) 758

39. V.L. Solozhenko, V.V. Chernyshev, G.V. Fetisov, V.B. Rybakov, I.A. Petrusha, J. Phys. Chem. Solids 51 (1990) 1011

40. A. San Miguel, Chem. Soc. Rev. 35 (2006) 876 


\section{Figures captions}

Figure 1: Experimental X-ray diffraction pattern $(+)$ compared to calculated one (solid line) using a Lebail model for a phase mixture of cubic (IMode) carbon nitride (upper ticks) and Re originating from the gasket of the open DAC (lower ticks). The residual is also shown (lower solid line).

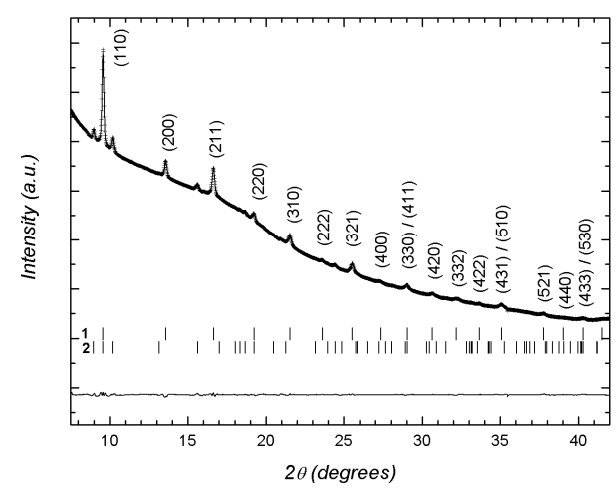

Figure 2: HRTEM micrograph of nanocrystalline domains embedded in an amorphous matrix. Digital diffractogram and zoom of the HRTEM image of the nanodomain labelled 1 are presented on upper and lower inserts, respectively. 


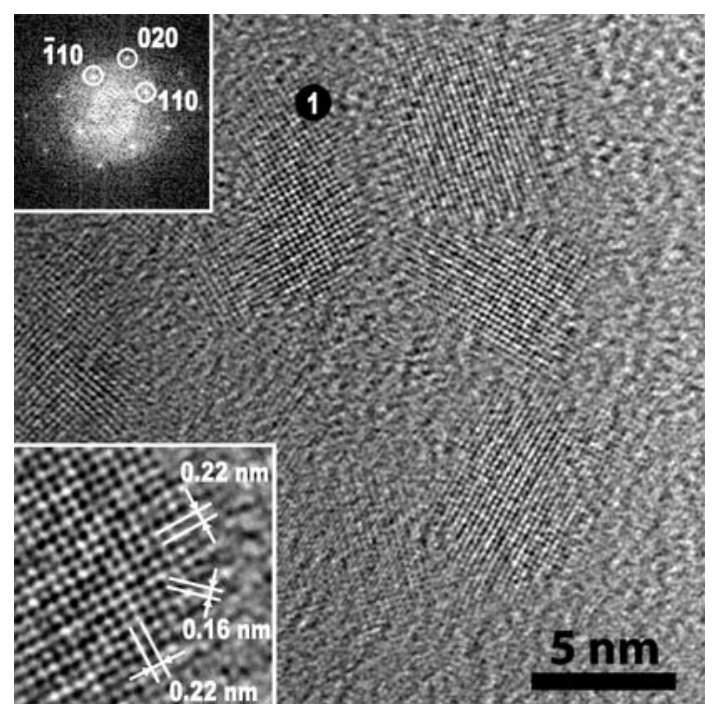

Figure 3: $\quad$ Chemical characterization of the $20-50 \mathrm{~nm}$ nanoparticles. HRTEM micrograph (a) and EFTEM ( $\mathrm{K}$ edge of $\mathrm{N}$ ) image (b) of nanocrystalline domains embedded in an amorphous matrix are shown. The new crystalline phase clearly shows higher $\mathrm{N}$ concentrations (red areas) than the amorphous matrix.
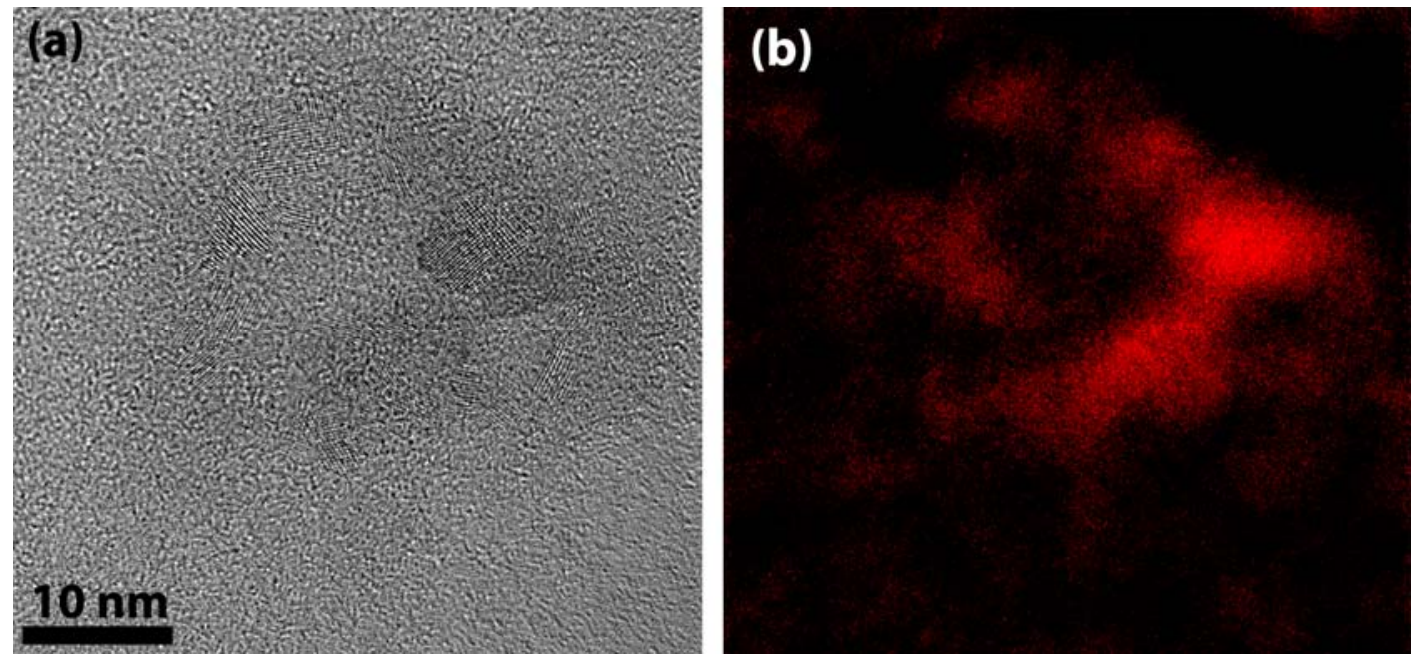
Figure 4: Experimental compression curve measured at $300 \mathrm{~K}$ for diamond (close circles) and cubic carbon nitride (open circle) together with fits using the third-order Birch-Murnaghan (BM) equation of state (solid lines). Bulk modulus of $355 \mathrm{GPa}\left(\mathrm{B}_{0}^{\prime}=3.2\right)$ for $\mathrm{c}-\mathrm{CN}_{\mathrm{x}}$ indicates its impressive low compressibility.

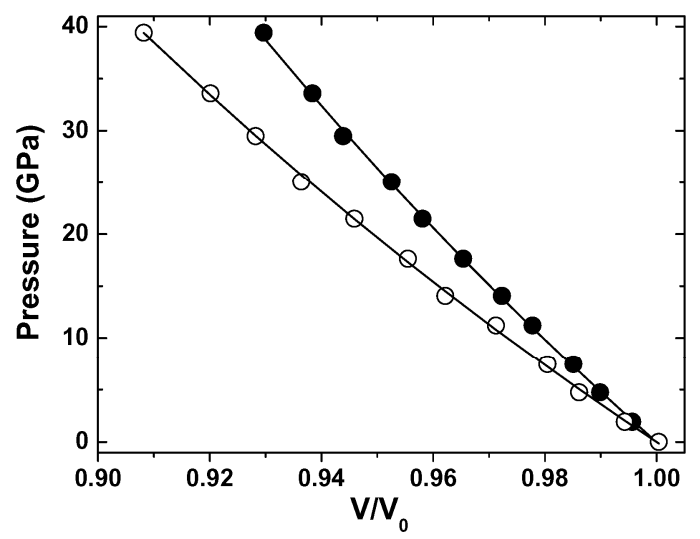

